\title{
FUZZY METRIC SPACE AND APPLICATIONS IN IMAGE PROCESSING
}

\author{
NEBOJŠA M. RALEVIĆ ${ }^{*}$, MARIJA PAUNOVIĆ ${ }^{2}$, AND BRATISLAV IRIČANIN ${ }^{3,4}$ \\ ${ }^{1}$ University of Novi Sad, Faculty of Technical Sciences, \\ Department of Fundamental Sciences. Novi Sad, Serbia \\ ${ }^{2}$ University of Kragujevac, Faculty of Hotel Management and Tourism in Vrnjačka Banja. \\ Vrnjačka Banja, Serbia \\ ${ }^{3}$ University of Beograd, School of Electrical Engineering. Belgrade, Serbia \\ ${ }^{4}$ University of Kragujevac, Faculty of Mechanical and Civil Engineering in Kraljevo, Kraljevo, Serbia \\ *Corresponding author. E-mail: nralevic@uns.ac.rs
}

\section{DOI:10.20948/mathmontis-2020-48-9}

Summary. In this paper, the notions of fuzzy T-metric and fuzzy S-metric have been introduced, and then, examples of known fuzzy metrics are provided, as well as theorems that enable algorithms for putting up new metrics. Recently, there has been renewed interest in some of their properties, which are further shown, these being polygonal inequality, and two new classes of functions have been shown to be regarded as fuzzy metric. By applying these fuzzy metrics, an algorithm has been used in order to remove image noise. The goal was to improve the sharpness and the quality of the image, which is expressed and measured by means of the image quality index UIQI. It has been shown that the general image, which is filtered by this algorithm, has greater sharpness than the image filtered by the median filter, which is probably the most commonly used vector filter.

\section{INTRODUCTION}

There is evidence that the notion of a probabilistic metric space plays a crucial role in regulating a generalization of metric space in which the distance between $x$ and $y$ is replaced by the distribution function $F_{x, y}$ where the value of that distribution function at some point $a$ is interpreted as the probability in which case, the distance between $x$ and $y$ is smaller of $a$. A key aspect of and the introduction of the concept of fuzzy set was proposed by L. Zadeh in 1965.

In recent years, there has been an increasing interest in fuzzy logic and recent developments in fuzzy sets have heightened the need for initiating wider development of the theory of fuzzy logic and fuzzy sets, as well as other mathematical fields where they were meant to replace standard sets. Characterization of fuzzy sets is important for our increased understanding of fuzzy logic and its consequences, and in this manner the theory of fuzzy metric spaces has evolved, where the distance between elements $x$ and $y$ is a fuzzy set with certain properties. There are several important areas where this theory makes an original contribution to finding increasing application particularly to specific practical problems one of which is image processing.

The whole concept of thinking that a distance between two objects is no longer a classical, but a fuzzy set, has led to further development of theoretical parts of mathematics in which the has term appeared, as well as the application of a part of this knowledge in various engineering sciences, even in medicine' sciences. Perhaps the best example is provided by the functional analysis, where the counterparts are defined by the classical notions of a sphere, 
neighborhood, convergence, etc., in fuzzy metric spaces. It is in this manner that the theory of a fixed point in the complete fuzzy metric spaces is considered as a natural continuation of research in this field in probabilistic metric spaces (see e.g. [2]).

Practical problems in which distance was used and their solution with fuzzy distance have obtained a new meaning of problem interpretation and the way of solving it. This is perhaps best seen in shape recognition, shape analysis and image processing, which have direct applications in practice. For example, classifying data into clusters, in which case each of them contains objects having the maximum of similarity, i.e. the minimum distance between each other, with as many objects from other clusters as possible.

Image processing and the problems considered in this area, such as, for example, filtering and segmentation of the image, abundantly use the distance in terms of similarities, i.e. differences of images, i.e. parts of images, and the "ambiguity" itself directly indicates the need for fuzzy distances.

The paper is organized as follows. The second section presents the basic notions of $t$-norm, $t$-conorms, fuzzy complements and their properties which will be required when proving the properties of fuzzy metrics (see [9], [10]). In the third section, the fuzzy $S$-metric and the fuzzy $T$-metric are defined and the examples relevant to the application are provided. Polygonal inequality has also been proven and two new classes of set functions have been defined, and the findings should make an important contribution to the field of fuzzy metrics. The properties by which new fuzzy metrics can be defined from existing classes are presented. Evidence suggests that fuzzy metric parameter values are among the most important factors for enabling selection of the metric that further offers the best performance in image filtering. In the section 4, the values of the described parameters are further experimentally determined. Ultimately, the main aim of this study is to investigate the differences between the quality of image filtering, utilizing our algorithm and filtering using VMF both of which, were calculated by means of the UIQI metric, which has been defined in [21], and they are subsequently compared.

\section{PRELIMINARIES}

This paper sets out to assess the effects of triangular norms and conorms and thus, definitions of triangular norms and conorms and some of their properties are taken into consideration and provided in the references (see [9], [10]).

Definition 2.1. For binary operation $N:[0,1]^{2} \rightarrow[0,1]$ which satisfies the following axioms for all $a, b, c, d \in[0,1]$ :

$\left.n_{1}\right) N(a, e)=a$ (boundary condition);

$\left.n_{2}\right) c \leq d \Rightarrow N(a, c) \leq N(a, d)$ ( monotonicity);

$\left.n_{3}\right) N(a, b)=N(b, a)$ ( commutativity);

$\left.n_{4}\right) N(a, N(b, c))=N(N(a, b), c)$ ( associativity).

we say that $N$ is norm.

If $e=1$, then $N$ is the triangular norm (shorter $t$-norm), and instead of $N$ we write $T$. If $e=$ 0 , then $N$ is the triangular conorm (shorter $t$-conorm), and instead of $N$ we write $S$.

A norm $N$, is an Archimedean norm if $N$ is continuous and for all $a \in(0,1), N(a, a)<a$ for $t$-norm $N$ and for all $a \in(0,1), N(a, a)>a$ for $t$-conorm $N$.

As $b \leq 1, T(a, b) \leq T(a, 1)=a$, and similarly $T(a, b) \leq b$, imply 


$$
T(a, b) \leq \min \{a, b\} .
$$

As $b \geq 0, S(a, b) \geq S(a, 0)=a$, and similarly $S(a, b) \geq b$, imply

$$
S(a, b) \geq \max \{a, b\} \text {. }
$$

Remark 1. From the conditions given in the definition of the norm follows the monotonicity by coordinates, i.e. for all $a_{1}, a_{2}, b_{1}, b_{2} \in[0,1]$

$$
a_{1} \leq a_{2} \wedge b_{1} \leq b_{2} \Rightarrow N\left(a_{1}, b_{1}\right) \leq N\left(a_{2}, b_{2}\right) .
$$

It is necessary here to clarify exactly what is meant by the monotonic condition replacement. Namely, replacing the monotonic condition in Definition 2.1 by condition (3), an equivalent definition of the norm is obtained.

If, in the definition of the norm, instead of the axiom of monotonicity, a strict monotonicity is valid, i.e.

$$
a_{1}<a_{2} \wedge b_{1}<b_{2} \Rightarrow N\left(a_{1}, b_{1}\right)<N\left(a_{2}, b_{2}\right),
$$

for all $a_{1}, a_{2}, b_{1}, b_{2} \in[0,1]$, then the norm is strict.

Definition 2.2. A decreasing generator $g$ is a continuous strictly decreasing function from $[0,1]$ to $\mathbb{R}$, such that $g(1)=0$.

An increasing generator $g$ is a continuous strictly increasing function from $[0,1]$ to $\mathbb{R}$, such that $g(0)=0$.

Definition 2.3. The power of the norm is given by formulas:

$$
N^{1}\left(a_{1}, a_{2}\right)=N\left(a_{1}, a_{2}\right), N^{n}\left(a_{1}, \ldots, a_{n}, a_{n+1}\right)=N\left(N^{n-1}\left(a_{1}, \ldots, a_{n}\right), a_{n+1}\right)(n \geq 2) .
$$

Remark 2. If $T$ is a $t$-norm, then:

$$
\begin{gathered}
T\left(a_{1}, a_{2}\right)=1 \Leftrightarrow a_{1}=a_{2}=1, \\
T^{n}\left(a_{1}, a_{2}, \ldots, a_{n+1}\right)=1 \Leftrightarrow a_{1}=\ldots=a_{n+1}=1 .
\end{gathered}
$$

Remark 3. If $T$ is a strict $t$-norm, then:

$$
\begin{gathered}
T\left(a_{1}, a_{2}\right)=0 \Leftrightarrow a_{1}=0 \vee a_{2}=0, \\
T^{n}\left(a_{1}, a_{2}, \ldots, a_{n+1}\right)=0 \Leftrightarrow a_{1}=0 \vee \ldots \vee a_{n+1}=0 .
\end{gathered}
$$

Remark 4. If $S$ is a $t$-conorm, then:

$$
\begin{gathered}
S\left(a_{1}, a_{2}\right)=0 \Leftrightarrow a_{1}=a_{2}=0 . \\
S^{n}\left(a_{1}, a_{2}, \ldots, a_{n+1}\right)=0 \Leftrightarrow a_{1}=\ldots=a_{n+1}=0 .
\end{gathered}
$$

Remark 5. If $S$ is a strict $t$-conorm, then:

$$
\begin{gathered}
S\left(a_{1}, a_{2}\right)=1 \Leftrightarrow a_{1}=1 \vee a_{2}=1 . \\
S^{n}\left(a_{1}, a_{2}, \ldots, a_{n+1}\right)=1 \Leftrightarrow a_{1}=1 \vee \ldots \vee a_{n+1}=1 .
\end{gathered}
$$


Definition 2.4. The function $c:[0,1] \rightarrow[0,1]$ is a fuzzy complement, if following conditions are satisfied:

$\left.c_{1}\right) c(0)=1$ i $c(1)=0$, (boundary conditions)

$\left.c_{2}\right)(\forall a, b \in[0,1]) a \leq b \Rightarrow c(a) \geq c(b)$ (monotonicity).

If $c(c(a))=a$ holds for all $a \in[0,1]$, then a function $c$ is involutive.

If $c$ is a continuous function, then we say that $c$ is a continuous fuzzy complement.

If $c:[0,1] \rightarrow[0,1]$ is an involutive monotonic non-increasing function, it follows that $c$ is a continuous bijective function for which boundary conditions are valid (see [10]).

The triangular norm $T$ and the triangular conorm $S$ are dual with respect to the fuzzy complement $c$ if and only if

$$
c(T(a, b))=S(c(a), c(b)) \text { and } c(S(a, b))=T(c(a), c(b)) .
$$

$(T, S, c)$ is called a dual triple.

For the triangular norm $T$ and the involutive fuzzy complement $c$, the binary operation $S$ on $[0,1]$ defined with

$$
S(a, b)=c(T(c(a), c(b)))
$$

for all $a, b \in[0,1]$, is a triangular conorm $S$ such that $(T, S, c)$ is a dual triple.

\section{FUZZY METRICS}

This section presents the findings of the research, focusing on the fuzzy $S$-metric and the fuzzy $T$-metric, which are being considered. Some of the new characteristics of the $T$-fuzzy metrics are presented. The main issues addressed in this section of the paper refer to the well known notions and characteristics and in the literature, these terms tend to be used to refer to the notions given and proven in the papers [6], [7], and [19].

Definition 3.1. [19] Let $X \neq \emptyset$,

i) $S$ be a continuous $t$ - conorm,

ii) $T$ be a continuous $t$ - norm,

and $\mathbf{d}$ is a fuzzy set defined on $X \times X \times(0,+\infty)$, that satisfies the following conditions for all $x, y, z \in X, \alpha, \beta>0$ :

(1) i) $\mathbf{d}(x, y, \alpha) \in[0,1)$,ii) $\mathbf{d}(x, y, \alpha) \in(0,1]$;

(2) i) $\mathbf{d}(x, y, \alpha)=0 \Leftrightarrow x=y$, ii) $\mathbf{d}(x, y, \alpha)=1 \Leftrightarrow x=y$;

(3) i), ii) $\mathbf{d}(x, y, \alpha)=\mathrm{d}(y, x, \alpha)$;

(4) i) $S(\mathbf{d}(x, y, \alpha), \mathbf{d}(y, z, \beta)) \geq \mathbf{d}(x, z, \alpha+\beta)$, ii) $T(\mathbf{d}(x, y, \alpha), \mathbf{d}(y, z, \beta)) \leq \mathbf{d}(x, z, \alpha+\beta)$;

(5) i), ii) $\mathbf{d}(x, y,-):(0,+\infty) \rightarrow[0,1]$ is a continuous function.

The fuzzy set $\mathbf{d}$ is called

i) a fuzzy $S$-metric and a triple $(X, \mathbf{d}, S)$ is fuzzy $S$-metric space (where d satisfies (1-5)i)) ;

ii) a fuzzy $T$-metric and a triple $(X, \mathbf{d}, T)$ is fuzzy $T$-metric space (where $\mathbf{d}$ satisfies (1-5) ii)).

If instead of (1), it holds that $\mathbf{d}(x, y, \alpha) \in[0,1]$, the fuzzy set $\mathbf{d}$ is a fuzzy $S$-metric (fuzzy $T$-metric) in the broader sense, and $(X, \mathbf{d}, S)((X, \mathbf{d}, T))$ is a fuzzy $S$-metric (fuzzy $T$-metric) space in the broader sense.

We will mark the fuzzy $S$-metric with $\mathbf{s}$ and the fuzzy $T$-metric with $\mathbf{t}$, and we will write 
the mark $\mathbf{d}$ if some statement is valid in both cases and use the term fuzzy metric.

Definition 3.2. [19] Fuzzy metric $\mathbf{d}$ is stationary on $X$ if $\mathbf{d}$ does not depend of $\alpha$, i.e. if for all fixed $x, y \in X$, the function $\mathbf{d}_{x, y}(\alpha)=\mathbf{d}(x, y, \alpha)$ is a constant.

Remark 6. Fuzzy $S-$ metric $\mathbf{s}(x, y,-):(0,+\infty) \rightarrow[0,1]$ is non-decreasing function, and fuzzy $T-$ metric $\mathbf{t}(x, y,-):(0,+\infty) \rightarrow[0,1]$ is non-increasing function.

Putting that $y=z$ in the triangle inequality

$$
S(\mathbf{s}(x, y, \alpha), \mathbf{s}(y, y, \beta))=S(\mathbf{s}(x, y, \alpha), 0)=\mathbf{s}(x, y, \alpha) \geq \mathbf{s}(x, y, \alpha+\beta),
$$

supposing $0<\alpha_{1}<\alpha_{2}$, for $\alpha=\alpha_{1}$, and $\beta=\alpha_{2}-\alpha_{1}$, imply $\mathbf{s}\left(x, y, \alpha_{1}\right) \geq \mathbf{s}\left(x, y, \alpha_{2}\right)$, i.e. $\mathbf{s}(x, y,-)$ is non-increasing function.

Putting that $y=z$ in the triangle inequality

$$
T(\mathbf{t}(x, y, \alpha), \mathbf{t}(y, y, \beta))=T(\mathbf{t}(x, y, \alpha), 1)=\mathbf{t}(x, y, \alpha) \leq \mathbf{t}(x, y, \alpha+\beta),
$$

supposing $0<\alpha_{1}<\alpha_{2}$, for $\alpha=\alpha_{1}$, and $\beta=\alpha_{2}-\alpha_{1}$, imply $\mathbf{t}\left(x, y, \alpha_{1}\right) \leq \mathbf{t}\left(x, y, \alpha_{2}\right)$, i.e. $\mathbf{t}\left(x, y,{ }_{-}\right)$is non-decreasing function.

Remark 7. The triangle inequality, is trivial satisfied if $x=y$ or $y=z$ or $x=z$.

Indeed, for example (4) i)

$$
\begin{gathered}
x=z \Rightarrow S(\mathbf{d}(x, y, \alpha), \mathbf{d}(y, x, \beta)) \geq \mathbf{d}(x, x, \alpha+\beta)=0 \Leftrightarrow \top, \\
x=y \Rightarrow S(\mathbf{d}(x, x, \alpha), \mathbf{d}(x, z, \beta))=S(0, \mathbf{d}(x, z, \beta))=\mathbf{d}(x, z, \beta) \geq \mathbf{d}(x, z, \alpha+\beta) \Leftrightarrow \top, \\
y=z \Rightarrow S(\mathbf{d}(x, y, \alpha), \mathbf{d}(y, y, \beta))=S(\mathbf{d}(x, y, \alpha), 0)=\mathbf{d}(x, y, \alpha) \geq \mathbf{d}(x, y, \alpha+\beta) \Leftrightarrow \top .
\end{gathered}
$$

Theorem 3.1. If $\mathbf{d}: X \times X \times(0,+\infty) \rightarrow \mathrm{R}$ is a fuzzy $T$-metric with respect to the norm $T$, then polygonal inequality, for $n \geq 2$, hold:

$$
T^{n-1}\left(\mathbf{d}\left(x_{1}, x_{2}, \alpha_{1}\right), \mathbf{d}\left(x_{2}, x_{3}, \alpha_{2}\right), \ldots, \mathbf{d}\left(x_{n}, x_{n+1}, \alpha_{n}\right)\right) \leq \mathbf{d}\left(x_{1}, x_{n+1}, \alpha_{1}+\alpha_{2}+\ldots+\alpha_{n}\right) .
$$

For case $S-$ metric with respect to the conorm $S$, for $n \geq 2$, hold:

$$
S^{n-1}\left(\mathbf{d}\left(x_{1}, x_{2}, \alpha_{1}\right), \mathbf{d}\left(x_{2}, x_{3}, \alpha_{2}\right), \ldots, \mathbf{d}\left(x_{n}, x_{n+1}, \alpha_{n}\right)\right) \geq \mathbf{d}\left(x_{1}, x_{n+1}, \alpha_{1}+\alpha_{2}+\ldots+\alpha_{n}\right) \text {. }
$$

Proof. Let us show the polygonal inequality for the case of the fuzzy $T$-metric. It is analogous to the fuzzy $S$-metric as well. If $n=2$, i.e.,

$$
T\left(\mathbf{d}\left(x_{1}, x_{2}, \alpha_{1}\right), \mathbf{d}\left(x_{2}, x_{3}, \alpha_{2}\right)\right) \leq \mathbf{d}\left(x_{1}, x_{3}, \alpha_{1}+\alpha_{2}\right),
$$

statement is valid, because axiom (4)ii). Suppose the claim holds for $n=k$ and prove it for $n=$ $k+1$ :

$$
\begin{aligned}
& T^{k-1}\left(\mathbf{d}\left(x_{1}, x_{2}, \alpha_{1}\right), \mathbf{d}\left(x_{2}, x_{3}, \alpha_{2}\right), \ldots, \mathbf{d}\left(x_{k}, x_{k+1}, \alpha_{k}\right)\right) \leq \mathbf{d}\left(x_{1}, x_{k+1}, \alpha_{1}+\alpha_{2} \ldots+\alpha_{k}\right) \\
& \Rightarrow T^{k}\left(\mathbf{d}\left(x_{1}, x_{2}, \alpha_{1}\right), \mathbf{d}\left(x_{2}, x_{3}, \alpha_{2}\right), \ldots, \mathbf{d}\left(x_{k}, x_{k+1}, \alpha_{k}\right), \mathbf{d}\left(x_{k+1}, x_{k+2}, \alpha_{k+1}\right)\right) \\
& =T\left(T^{k-1}\left(\mathbf{d}\left(x_{1}, x_{2}, \alpha_{1}\right), \mathbf{d}\left(x_{2}, x_{3}, \alpha_{2}\right), \ldots, \mathbf{d}\left(x_{k}, x_{k+1}, \alpha_{k}\right)\right), \mathbf{d}\left(x_{k+1}, x_{k+2}, \alpha_{k+1}\right)\right) \\
& \leq T\left(\mathbf{d}\left(x_{1}, x_{k+1}, \alpha_{1}+\alpha_{2}+\ldots+\alpha_{k}\right), \mathbf{d}\left(x_{k+1}, x_{k+2}, \alpha_{k+1}\right)\right) \\
& \leq d\left(x_{1}, x_{k+2}, \alpha_{1}+\alpha_{2}+\ldots+\alpha_{k}+\alpha_{k+1}\right) .
\end{aligned}
$$


Theorem 3.2. [19] If $(X, \mathbf{s}, S)$ is a fuzzy $S$-metric space and the $T$ is a $t$-norm dual to the $t$-conorm $S$ with respect to the continuous involutive fuzzy complement $c$, then $(X, c \circ \mathbf{s}, T)$ is a fuzzy $T$-metric space.

If $(X, \mathbf{t}, T)$ is a fuzzy $T$-metric space and $S$ is a $t$-conorm dual to the norm $T$ with respect to a continuous involutive fuzzy complement $c$, then $(X, c \circ \mathbf{t}, S)$ is a fuzzy $S$-metric space.

The theorem is also valid for fuzzy metric spaces in the broader sense.

Example 1. [6, 19] The mapping $\mathbf{t}_{K}: \mathbb{R}^{+} \times \mathbb{R}^{+} \rightarrow \mathbb{R}$ defined by $\mathbf{t}_{K}(x, y)=\frac{\min \{x, y\}+K}{\max \{x, y\}+K}$, where $K>0$, is a fuzzy $T$-metric with respect to multiplication, and $\mathbf{s}_{K}(x, y)=\frac{|x-y|}{\max (x, y)+K}$ is a fuzzy $S$-metric with respect to the algebraic sum, $S(x, y)=1-(1-x)(1-y)=x+y-x y$, dual to $T$ with respect to the standard fuzzy complement.

Example 2. Let $f: X \rightarrow \mathbb{R}^{+}$be one-to-one function and let $g: \mathbb{R}^{+} \rightarrow[0, \infty]$ be an increasing continuous function. The mapping $\mathbf{t}: X \times X \times \mathbb{R}^{+} \rightarrow[0,1]$ defined by

$$
\mathbf{t}(x, y, \alpha)=\left(\frac{\frac{(f(x))^{p}+(f(y))^{p}}{2}+g(\alpha)}{(\max \{f(x), f(y)\})^{p}+g(\alpha)}\right)^{q},
$$

where $p, q>0$ fixed, is a fuzzy $T$-metric with respect to multiplication.

Proof. $f(x), f(y) \in \mathbb{R}^{+}, g(\alpha) \geq 0$. Without loss of generality, let $f(x) \leq f(y)$. Then

$$
\begin{aligned}
& (f(x))^{p} \leq(f(y))^{p} \text {, i.e., } \\
& (f(x))^{p}+(f(y))^{p} \leq(f(y))^{p}+(f(y))^{p}=2(f(y))^{p}=2 \max \left\{(f(x))^{p},(f(y))^{p}\right\} \\
& \Rightarrow \frac{(f(x))^{p}+(f(y))^{p}}{2} \leq \max \left\{(f(x))^{p},(f(y))^{p}\right\} \\
& \Rightarrow 0<\frac{(f(x))^{p}+(f(y))^{p}}{2}+g(\alpha) \leq \max \left\{(f(x))^{p},(f(y))^{p}\right\}+g(\alpha) \\
& \Rightarrow 1 \geq \mathbf{t}(x, y, \alpha)=\left(\frac{\frac{(f(x))^{p}+(f(y))^{p}}{2}+g(\alpha)}{\max \left\{(f(x))^{p},(f(y))^{p}\right\}+g(\alpha)}\right)^{q}>0 . \\
& (\Leftarrow) x=y \Rightarrow \mathbf{t}(x, y, \alpha)=\left(\frac{\frac{(f(x))^{p}+(f(y))^{p}}{2}+g(\alpha)}{\max \left\{(f(x))^{p},(f(y))^{p}\right\}+g(\alpha)}\right)^{q}=\left(\frac{(f(x))^{p}+g(\alpha)}{(f(x))^{p}+g(\alpha)}\right)^{q}=1 \\
& \left(\Rightarrow \mathbf{t}(x, y, \alpha)=\left(\frac{\frac{(f(x))^{p}+(f(y))^{p}}{2}+g(\alpha)}{\max \left\{(f(x))^{p},(f(y))^{p}\right\}+g(\alpha)}\right)^{q}=1 \Leftrightarrow \frac{(f(x))^{p}+(f(y))^{p}}{2}+g(\alpha)=\right. \\
& \max \left\{(f(x))^{p},(f(y))^{p}\right\}+g(\alpha) \Leftrightarrow(f(x))^{p}+(f(y))^{p}=2 \max \left\{(f(x))^{p},(f(y))^{p}\right\}:
\end{aligned}
$$


$f(x) \geq f(y) \Rightarrow(f(x))^{p}+(f(y))^{p}=2(f(x))^{p} \Rightarrow(f(y))^{p}=(f(x))^{p} \Rightarrow f(y)=f(x) \Rightarrow$ $y=x$

$f(x) \leq f(y) \Rightarrow(f(x))^{p}+(f(y))^{p}=2(f(y))^{p} \Rightarrow(f(x))^{p}=(f(y))^{p} \Rightarrow f(x)=f(y) \Rightarrow$ $x=y$ ( $f$ is one-to-one function).

$\mathbf{t}(x, y, \alpha)=\left(\frac{\frac{(f(x))^{p}+(f(y))^{p}}{2}+g(\alpha)}{(\max \{f(x), f(y)\})^{p}+g(\alpha)}\right)^{q}=\left(\frac{\frac{(f(y))^{p}+(f(x))^{p}}{2}+g(\alpha)}{(\max \{f(y), f(x)\})^{p}+g(\alpha)}\right)^{q}=\mathbf{t}(y, x, \alpha)$.

Let's prove inequality

$$
\begin{aligned}
& \mathbf{t}(x, y, \alpha) \cdot \mathbf{t}(y, z, \alpha) \leq \mathbf{t}(x, z, \alpha) . \\
& \text { (7) } \Leftrightarrow\left(\frac{\frac{(f(x))^{p}+(f(y))^{p}}{2}+g(\alpha)}{(\max \{f(x), f(y)\})^{p}+g(\alpha)}\right)^{q} \cdot\left(\frac{\frac{(f(y))^{p}+(f(z))^{p}}{2}+g(\alpha)}{(\max \{f(y), f(z)\})^{p}+g(\alpha)}\right)^{q} \\
& \leq\left(\frac{\frac{(f(x))^{p}+(f(z))^{p}}{2}+g(\alpha)}{(\max \{f(x), f(z)\})^{p}+g(\alpha)}\right)^{q} \\
& \Leftrightarrow \frac{\frac{(f(x))^{p}+g(\alpha)+(f(y))^{p}+g(\alpha)}{2}}{\max \left\{(f(x))^{p}+g(\alpha),(f(y))^{p}+g(\alpha)\right\}} \cdot \frac{\frac{(f(y))^{p}+g(\alpha)+(f(z))^{p}+g(\alpha)}{2}+g(\alpha)}{\max \left\{(f(y))^{p}+g(\alpha),(f(z))^{p}+g(\alpha)\right\}}
\end{aligned}
$$

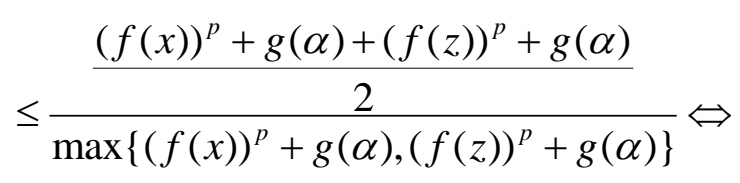

$$
\begin{aligned}
& \frac{X+Y}{\max \{X, Y\}} \cdot \frac{Y+Z}{\max \{Y, Z\}} \leq 2 \cdot \frac{X+Z}{\max \{X, Z\}},
\end{aligned}
$$

where $X=(f(x))^{p}+g(\alpha), Y=(f(y))^{p}+g(\alpha), Z=(f(z))^{p}+g(\alpha)$.

There are three cases: 1) $f(x) \leq f(y) \leq f(z)$, 2) $f(x) \leq f(z) \leq f(y)$,

and 3) $f(y) \leq f(x) \leq f(z)$, i.e., 1) $X \leq Y \leq Z$, 2) $X \leq Z \leq Y$, and 3) $Y \leq Z \leq Z$.

1) $(8) \Leftrightarrow \frac{X+Y}{Y} \cdot \frac{Y+Z}{Z} \leq 2 \frac{X+Z}{Z}$

$\Leftrightarrow(X+Y)(Z+Y) \leq 2(X+Z) Y$

$\Leftrightarrow Y^{2}+X Y+Z Y+X Z \leq 2 X Y+2 Z Y$

$\Leftrightarrow Y^{2}-(X+Z) Y+X Z \leq 0 \Leftrightarrow(Y-X)(Y-Z) \leq 0 \Leftrightarrow \top$.

The inequality is correct because two following inequalities hold: $X \leq Y$ and $Y \leq Z$.

2) $(8) \Leftrightarrow \frac{X+Y}{Y} \cdot \frac{Y+Z}{Y} \leq 2 \frac{X+Z}{Z}$

$\Leftrightarrow\left(Y^{2}+(X+Z) Y+X Z\right) Z \leq 2(X+Z) Y^{2}$ 


$$
\begin{aligned}
& \Leftrightarrow(X+Z) Z Y+X Z^{2} \leq 2 X Y^{2}+Z Y^{2} \\
& \Leftrightarrow X Z Y+Z^{2} Y+X Z^{2} \leq X Y^{2}+X Y^{2}+Z Y^{2} .
\end{aligned}
$$

This inequality is valid because:

$$
\begin{aligned}
& Z \leq Y \Rightarrow X Z Y \leq X Y^{2}, \\
& Z \leq Y \Rightarrow X Z^{2} \leq X Y^{2}, \\
& Z \leq Y \Rightarrow Z^{2} Y \leq Z Y^{2} .
\end{aligned}
$$

3) $(8) \Leftrightarrow \frac{X+Y}{X} \cdot \frac{Y+Z}{Z} \leq 2 \frac{X+Z}{Z} \Leftrightarrow(X+Y)(Y+Z) \leq 2 X \cdot(X+Z)$.

This inequality is true because the following inequalities hold:

$$
Y \leq X \Rightarrow X+Y \leq 2 X, Y \leq X \Rightarrow Y+Z \leq X+Z \text {. }
$$

The function $F(\alpha)=\left(\frac{a+g(\alpha)}{b+g(\alpha)}\right)^{q}$, where $a, b, g(\alpha)>0, a<b$, is monotonously increasing, because $g$ is monotonously increasing, so

$$
\begin{aligned}
& \mathbf{t}(x, y, \alpha) \leq \mathbf{t}(x, y, \alpha+\beta), \mathbf{t}(y, z, \beta) \leq \mathbf{t}(y, z, \alpha+\beta), \text { i.e., (7) implies: } \\
& \mathbf{t}(x, y, \alpha) \cdot \mathbf{t}(y, z, \beta) \leq \mathbf{t}(x, y, \alpha+\beta) \cdot \mathbf{t}(y, z, \alpha+\beta) \leq \mathbf{t}(x, z, \alpha+\beta) .
\end{aligned}
$$

The mapping $\mathbf{t}$ is obviously a continuous function, because $g$ is continuous function.

Special case of Example 2 is next example:

Example 3. [19] The mapping $\mathbf{t}_{K}: \mathbb{R}^{+} \times \mathbb{R}^{+} \rightarrow \mathbb{R}$ defined by $\mathbf{t}_{K}(x, y)=\frac{\frac{x+y}{2}+K}{\max \{x, y\}+K}$, where $K>0$, is a fuzzy $T$-metric with respect to multiplication, and $\mathbf{s}_{K}(x, y)=\frac{|x-y|}{2(\max (x, y)+K)}$ is the fuzzy $S$-metric with respect to the algebraic sum, dual to $T$ with respect to standard fuzzy complement.

Example 4. [19] The mapping $\mathbf{t}_{p}: \mathbb{R}^{+} \times \mathbb{R}^{+} \rightarrow \mathbb{R}, \quad p>0$ defined by

$$
\mathbf{t}_{p}(x, y)=\frac{\sqrt[p]{\frac{x^{p}+y^{p}}{2}}}{\max \{x, y\}},
$$

is a fuzzy $T$-metric with respect to multiplication.

Example 5. [6] Let $g: \mathbb{R}^{+} \rightarrow[0, \infty]$ be an increasing continuous function. If $(X, d)$ is a metric space then the mapping $\mathbf{t}: X \times X \times \mathbb{R}^{+} \rightarrow \mathbb{R}$ defined by

$$
\mathbf{t}(x, y, t)=\frac{g(t)}{g(t)+d(x, y)}
$$

is a fuzzy $T$-metric with respect to the multiplication. Specially, for $f(\alpha)=\alpha$ : 


$$
\mathbf{t}(x, y, t)=\frac{t}{t+d(x, y)}
$$

and its dual (with respect to the standard fuzzy complement) $\mathbf{s}(x, y, t)=1-\mathbf{t}(x, y, t)=\frac{d(x, y)}{t+d(x, y)}$ is a fuzzy $S$-metric with respect to the algebraic sum.

Theorem 3.3. Let $f: X \rightarrow(0,1]$ be one-to-one function and let $g: \mathbb{R}^{+} \rightarrow(0,1]$ be an increasing continuous function. The mapping $d: X \times X \times(0,+\infty) \rightarrow \mathbb{R}$ defined by

$$
\mathbf{d}(x, y, \alpha)=\left\{\begin{array}{ll}
1, & x=y \\
T(T(f(x), f(y)), g(\alpha)), & x \neq y
\end{array}=\left\{\begin{array}{ll}
1, & x=y \\
T^{2}(f(x), f(y), g(\alpha)), & x \neq y
\end{array},\right.\right.
$$

is a fuzzy T-metric with respect to the continuous $t$-norm $T$.

Proof. From Remark 3 follows

$\mathbf{d}(x, y, \alpha)=0 \Leftrightarrow T(f(x), f(y))=0 \vee g(\alpha)=0 \Leftrightarrow f(x)=0 \vee f(y)=0 \vee g(\alpha)=0 \Leftrightarrow \perp$, i.e. $\mathbf{d}(x, y, \alpha)=T(T(f(x), f(y)), g(\alpha)) \in(0,1]$;

Obviously, $x=y \Rightarrow \mathbf{d}(x, y, \alpha)=1$. Suppose that $x \neq y$ and $\mathbf{d}(x, y, \alpha)=1$, then from Remark $\mathbf{2}$ follows

$$
\begin{aligned}
& \mathbf{d}(x, y), \alpha)=T(T(f(x), f(y)), g(\alpha))=1 \Leftrightarrow T(f(x), f(y))=1 \wedge g(\alpha)=1 \\
& \Leftrightarrow f(x)=1 \wedge f(y)=1 \wedge g(\alpha)=1 \Rightarrow f(x)=f(y) \Rightarrow x=y . \text { Contradiction! } \\
& \mathbf{d}(x, y), \alpha)=T(T(f(x), f(y)), g(\alpha))=T(T(f(y), f(x)), g(\alpha))=\mathbf{d}(y, x, \alpha) ;
\end{aligned}
$$

From associativity of $t$-norm $T$, for $x \neq y \neq z \neq x$ :

$$
\begin{aligned}
T(\mathbf{d}(x, z, \alpha), \mathbf{d}(z, y, \beta)) & =T(T(T(f(x), f(z)), g(\alpha)), T(T(f(z), f(y)), g(\beta))) \\
& =T(T(f(x), f(z)), T(g(\alpha), T(T(f(z), f(y)), g(\beta)))) \\
& =T(T(f(x), f(z)), T(T(g(\alpha), T(f(z), f(y))), g(\beta))) \\
& =T(T(f(x), f(z)), T(T(T(f(z), f(y)), g(\alpha)), g(\beta))) \\
& =T(T(f(x), f(z)), T(T(f(z), f(y)), T(g(\alpha), g(\beta)))) \\
& =T(T(T(f(x), f(z)), T(f(z), f(y))), T(g(\alpha), g(\beta))) .
\end{aligned}
$$

From inequality (1), we have $T(f(x), f(z)) \leq f(x), T(f(z), f(y)) \leq f(y)$, so because of (3), it follows $T(T(f(x), f(z)), T(f(z), f(y))) \leq T(f(x), f(y))$.

From (1), and monotonicity of $T: T(g(\alpha), g(\beta)) \leq \min \{g(\alpha), g(\beta)\} \leq g(\alpha+\beta)$. Finally, 


$$
\begin{gathered}
T(\mathbf{d}(x, z, \alpha), \mathbf{d}(z, y, \beta))=T(T(T(f(x), f(z)), T(f(z), f(y))), T(g(\alpha), g(\beta))) \\
\leq T(T(f(x), f(y)), g(\alpha+\beta))=\mathbf{d}(x, y, \alpha+\beta) .
\end{gathered}
$$

Triangular norm $T$ and function $g$ are continuous, then $\mathbf{d}\left(x, y,,_{-}\right):(0,+\infty) \rightarrow[0,1]$ is a continuous function.

The following properties hold (see [19]) which enable construction of new fuzzy metrics.

Theorem 3.4. Let $\mathbf{d}$ be a stationary fuzzy metric (in the broader sense) with respect to the norm $N$. If $N$ is an Archimedean norm and $g$ its corresponding generator, then $d=g \circ \mathbf{d}$ is a standard metric.

Theorem 3.5. If $\mathbf{d}_{1}: X \times X \times(0,+\infty) \rightarrow[0,1)$ and $\mathbf{d}_{2}: X \times X \times(0,+\infty) \rightarrow[0,1)$ are fuzzy metrics, with respect to the strict norm $N$, then the mapping $\sigma\left(\mathbf{d}_{1}, \mathbf{d}_{2}\right): X \times X \times(0,+\infty) \rightarrow \mathbb{R}$ defined by $\sigma\left(\mathbf{d}_{1}, \mathbf{d}_{2}\right)(x, y, \alpha)=N\left(\mathbf{d}_{1}(x, y, \alpha), \mathbf{d}_{2}(x, y, \alpha)\right)$ is also a fuzzy metric with respect to the norm $N$. If $N$ is not a strict norm, then $\sigma\left(\mathbf{d}_{1}, \mathbf{d}_{2}\right)$ is a fuzzy metric in a broader sense.

Theorem 3.6. If $\mathbf{d}_{i}: X_{i} \times X_{i} \rightarrow[0,1), i=1, \ldots, n, n \in N$, are fuzzy metrics with respect to the strict norm $N$, then $\mathbf{d}: X^{2} \rightarrow[0,1], X=X_{1} \times \cdots \times X_{n}$ defined with

$$
\mathbf{d}(x, y)=N^{n-1}\left(\mathbf{d}_{1}\left(x_{1}, y_{1}\right), \mathbf{d}_{2}\left(x_{2}, y_{2}\right), \ldots, \mathbf{d}_{n}\left(x_{n}, y_{n}\right)\right), x=\left(x_{1}, \ldots, x_{n}\right), y=\left(y_{1}, \ldots, y_{n}\right),
$$

is the fuzzy metric with respect to the norm $N$. If $N$ is not a strict norm, then $\mathbf{d}$ is a fuzzy metric in a broader sense.

Example 6. If $\mathbf{t}_{i}: X_{i} \times X_{i} \rightarrow(0,1], i=1, \ldots, n, n \in \mathbb{N}$, are fuzzy $T$-metrics with respect to the product, then $\mathbf{t}: X^{2} \rightarrow(0,1], X=X_{1} \times \cdots \times X_{n}$ defined with

$$
\mathbf{t}(x, y)=\prod_{i=1}^{n} \mathbf{t}_{i}\left(x_{i}, y_{i}\right), x=\left(x_{1}, \ldots, x_{n}\right), y=\left(y_{1}, \ldots, y_{n}\right),
$$

is the fuzzy $T$-metric with respect to the product.

\section{APPLICATION}

In this section the application of fuzzy metrics in filtering colour images is given. The fuzzy metrics embodies a multitude of concepts that are influenced by image pixels. Each image pixel $\left(i, J_{i}\right)$ ("position", "colour") can be characterized by spatial coordinates of pixel $i_{1}$, $i_{2}$ (points $i=\left(i_{1}, i_{2}\right) \in I \times I, I=\{0,1, \ldots, n-1\}$ from the screen), and by vector $J_{i}=\left(J_{i}{ }^{1}, J_{i}{ }^{2}, J_{i}{ }^{3}\right)$, the first coordinate of which represents quantity of red colour, while the second coordinate is a quantity of green colour, and finally, the third one represents quantity of blue colour, these colour components being red, green, blue (RGB), respectively.

The assignment of image filtering is to replace the pixel which represents noise by pixel without noise, which can be achieved by replacing a central pixel $\left(\bar{i}_{i}, \bar{J}_{i}\right)$ in window 
$W=\left\{\left(i, J_{i}\right) \mid i \in I_{1} \times I_{2}\right\},\left(\bar{i}=\left(\bar{i}_{1}, \bar{i}_{2}\right) \in I_{1} \times I_{2}\right.$, and the size from window is an odd number $)$ with pixel that represents the other pixels from $W$ in the best possible way, i.e. by a pixel which is the most similar in colour and spatial distance to all the other pixels in $W$.

Selection bias is another potential concern because it is of enormous importance to pick up a good criterion for selecting such a pixel without noise, which will replace the pixel with noise in a given window $W$, because the choice of pixels affects the image quality, i.e. affects the degree of the removed noise.

A key issue is the safe disposal of criterion selection. Namely, the choice of a criterion will be conditioned by a good selection of fuzzy $T-$ metric c. All pixels in the some window $W$ a order relation will be induced by using metric c. This order relation will be used to compare pixels ("position", "colour") of the image and to choose a pixel that differs the least from all the other pixels in the window, i.e. which is the most similar to all other pixels in $W$ (regarding colour and distance). The central pixel in the given window $W$ will be replaced by the pixel found using the algorithm which is applied on each sliding window.

In the algorithm for filtering the image we use fuzzy $T$-metric $\mathbf{c}: W \times W \rightarrow \mathbb{R}$ defined with:

$$
\mathbf{c}\left(\left(i, J_{i}\right),\left(j, J_{j}\right)\right)=\mathbf{\tau}\left(J_{i}, J_{j}\right) \cdot \mathbf{t}(i, j) .
$$

Fuzzy $T$-metric which is used in order to measure similarity in colours among pixels is marked with $\tau$. It is defined in the following way:

$$
\boldsymbol{\tau}\left(J_{i}, J_{j}\right)=\boldsymbol{\tau}_{1}\left(J_{i}^{1}, J_{j}^{1}\right) \cdot \boldsymbol{\tau}_{2}\left(J_{i}^{2}, J_{j}^{2}\right) \cdot \boldsymbol{\tau}_{3}\left(J_{i}^{3}, J_{j}^{3}\right)=\prod_{l=1}^{3} \frac{\frac{J_{i}^{l}+J_{j}^{l}}{2}+K}{\max \left\{J_{i}^{l}, J_{j}^{l}\right\}+K} .
$$

Fuzzy $T$-metric that considers spatial distance between pixels is marked with $\mathbf{t}$. It is defined in the following way:

$$
\mathbf{t}(i, j)=\frac{t}{t+\sqrt{\left(i_{1}-j_{1}\right)^{2}+\left(i_{2}-j_{2}\right)^{2}}} .
$$

That the mappings $\boldsymbol{\tau}, \mathbf{t}$ and $\mathbf{c}$ are fuzzy metrics follows from the Examples 3 and 5 and Theorem 3.6.

The metric used for the comparison of the quality of images is UIQI, which is defined in [21]. Let $\mathbf{x}=\left\{x_{i} \mid i=1,2, \ldots, n\right\}$ and $\mathbf{y}=\left\{y_{i} \mid i=1,2, \ldots, n\right\}$ original and test image signals. Image quality index is defined by following formulas:

$$
Q=\frac{4 \sigma_{x y} \overline{x y}}{\left(\sigma_{x}^{2}+\sigma_{y}^{2}\right)\left[(\bar{x})^{2}+(\bar{y})^{2}\right]}
$$

where

$$
\begin{gathered}
\bar{x}=\frac{1}{n} \sum_{i=1}^{n} x_{i}, \quad \bar{y}=\frac{1}{n} \sum_{i=1}^{n} y_{i}, \\
\sigma_{x}^{2}=\frac{1}{n-1} \sum_{i=1}^{n}\left(x_{i}-\bar{x}\right)^{2}, \quad \sigma_{y}^{2}=\frac{1}{n-1} \sum_{i=1}^{n}\left(y_{i}-\bar{y}\right)^{2},
\end{gathered}
$$


Index can be write as:

$$
\sigma_{x y}=\frac{1}{n-1} \sum_{i=1}^{n}\left(x_{i}-\bar{x}\right)\left(y_{i}-\bar{y}\right)
$$

$$
Q=\frac{\sigma_{x y}}{\sigma_{x} \sigma_{y}} \cdot \frac{2 \overline{x y}}{(\bar{x})^{2}+(\bar{y})^{2}} \cdot \frac{2 \sigma_{x} \sigma_{y}}{\sigma_{x}^{2}+\sigma_{y}^{2}} .
$$

Of particular concern is the first component, which is the correlation coefficient between $x$ and $y$. Then, the second component shows how close are the pixel brightness values of $x$ and $y$. And, finally, the third component measures how similar are the contrasts between $x$ and $y$.

The particular metric of quality UIQI is based on the fact that every image distortion is viewed as a combination of three factors: loss of correlation, luminance distortion and contrast distortion.

For additional literature reading about fuzzy filtering, authors recommend the following list: [5], [13], [14], [15] and [20].

In the following example of image quality UIQI, the chosen size of window is 5 .

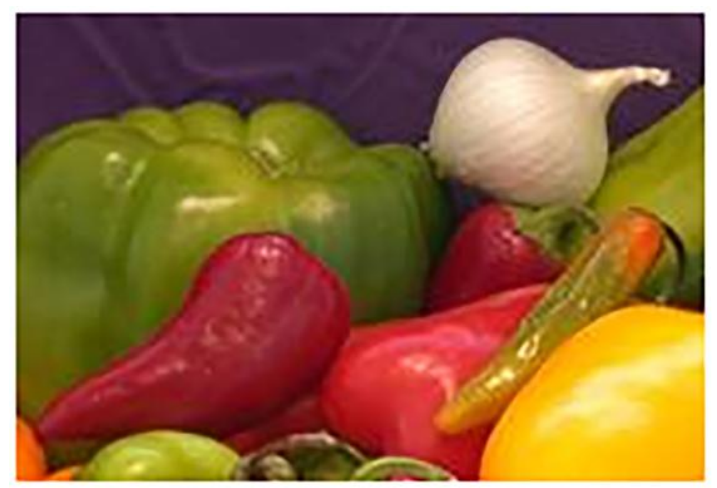

Figure 1. Onion, Original image in jpg format

As can be seen, the filtered image given below is contaminated with $10 \%$ salt and pepper noise.

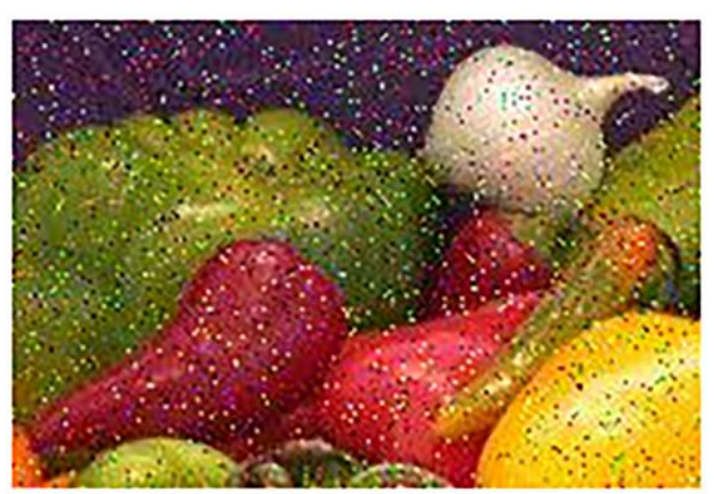

Figure 2. Onion, Salt and pepper noise $10 \%$ of noise

A much debated question is whether these metrics can be defined properly. The metric $\mathrm{c}$ which is defined with (12), where $\boldsymbol{\tau}$ and $\mathbf{t}$ are defined by (13) and (14), respectively. The 
values of metric for the image quality UIQI for each colour for the filtered image by applying the method proposed in this paper are equal to:

UIQI: [0.317457074736562,0.316259056738023,0.225750489132398].

The sharpness for image filtered by our metric is 0.9908 .

The values of metric of image quality UIQI for each colour for filtered image by median filter (see [1]) with window size three are equal to:

UIQI: [0.683856162855340,0.753420696483180,0.615343870586316].

The sharpness for image filtered by VMF is 0.4635 .

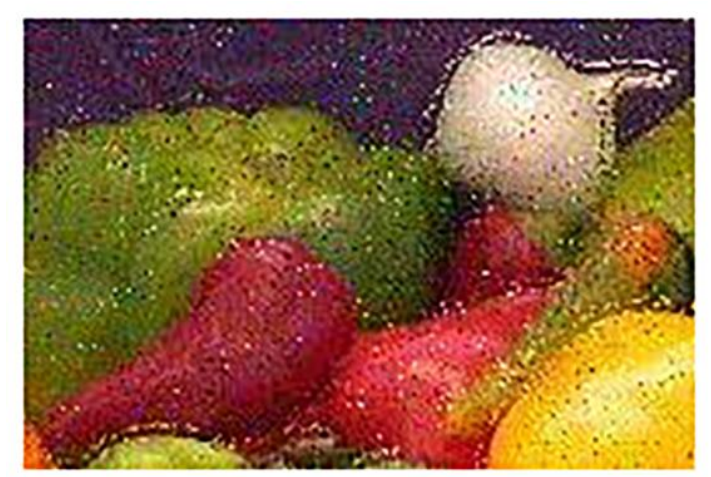

Figure 3. Onion, filtered image, onion $S \& P$ noise window size is $5, \mathrm{~K}=2250, \mathrm{t}=0.1$ fuzzy paper denoised armaks

The result was that our image has slightly lower values for corresponding UIQI image quality, but much higher sharpness. This will enhance the understanding the link between quality and sharpness. And this is very important in cases, in which details in the image itself are necessary to be properly. We have used for measuring sharpness image quality metrics introduced in [16].

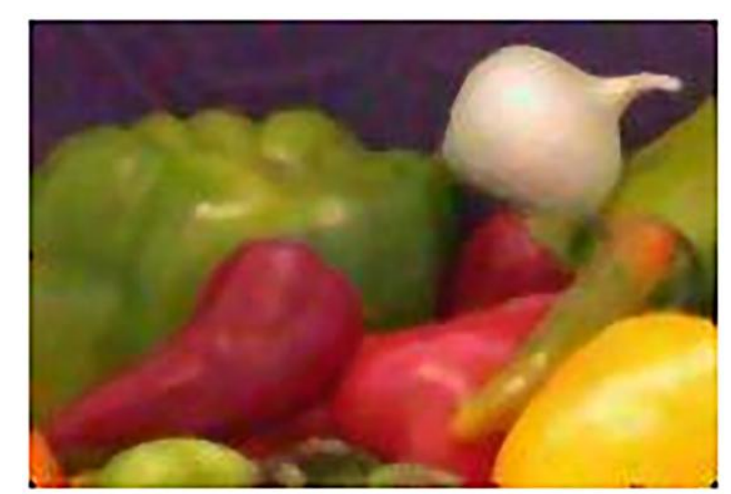

Figure 4. Onion, filtered by median filter, window size is 5; onion $S \& P$ noise MEDIAN denoised

In the articles [8] and [19], denoising was investigated on digital images of Lena and Baboon, using fuzzy metrics. In both cases, greater sharpness of the image was shown for the selected parameter values, and in the first case better UIQI. 


\section{CONCLUSIONS}

Distance is a term that is very commonly used both in mathematics and other sciences. The need for the notion of distance caused it to evolve depending on the nature of the set for which it is defined, and brought about the properties required to possess that particular mapping up to what constitutes its set of values. In this paper, the distance is defined over an abstract set, and the distance between its two elements is a fuzzy set (see e.g. $[17,18]$ ). The parameter that provides it with that particular fuzzy nature enable higher possibilities of application, as is the case here. The required properties are the following ones: boundedness, symmetry, some variants of the inequality of a triangle (in relation to the conorm, i.e. the norm), continuity according to the parameter, which all lead to the notions $\mathbf{s}$ and $\mathbf{t}$ fuzzy metrics. Historically, the idea started from K. Menger [12] and probabilistic spaces, where the distance between two elements was assessed by means of the distribution function. Subsequently, the notion of the fuzzy space was reached through the whole series of mathematicians, more specifically, in the researches of I. Kramosil, J. Michalek [11], up to A. George, P. Veramani [3, 4], and later on V. Gregory, A. Sapena [6, 7], and their associates. There is a huge number of scientific papers related to the topic of these spaces.

In this paper, the inequality of polygons for the considered fuzzy spaces has been proved, as well as new examples of those spaces, which would enable a wider scope of application of such metrics. One of these applications is image processing (image filtering, image segmentation, etc.). Accompanied by a suitable choice of phase distances, and by means of varying their appropriate parameters, we determine the best possible solution to our problem. The problem of image segmentation considered in this article is comprised of replacing the noisy pixel in the image with a better one, taking into account the influence of the surrounding pixels. The process of selecting a new pixel is influenced by both the spatial fuzzy distance of the pixels and the difference in the pixel brightness within the image in the RGB format, also measured by means of the selected fuzzy metric. The new fuzzy metric combines these two fuzzy metrics in the optimal manner into one evaluation for selecting a new pixel.

New research is aimed at searching for metrics that would perform better in their application, as well as at examining their properties and finding new possibilities of application in other areas.

Acknowledgements: First author acknowledge the financial support of the Ministry of Education, Science and Technological Development of the Republic of Serbia, in the frame of Projects applied under No. TR 34014 and No. ON 174009.

The work of third author was supported by the Serbian Ministry of Education, Science and Technological Development projects III 41025 and OI 171007.

The authors express their sincere gratitude to Prof. Miloš Đurić (ETF Belgrade, Serbia) for his careful reading of the paper in the stage when it was a manuscript and for his valuable comments that substantially improved the text.

\section{REFERENCES}

[1] J. Astola, P. Haavisto, and Y. Neuvo, "Vector median filters", Proceedings of the IEEE, 78(4), 678-689 (1990). doi 10.1109/554807

[2] Lj. Gajić, N. M. Ralević, and D. Karaklić, "Prostori sa fazi rastojanjem", META 2019, The Fourth Conference on Mathematics in Engineering: Theory and Applications, Faculty of Technical Sciences, May 10-12 ${ }^{\text {th }}$, 2019, str. 85-90, Novi Sad, Serbia. 
[3] A. George and P. Veeramani P., On some results in fuzzy metric spaces. Fuzzy Sets Syst., 64(3), 395-399 (1994), doi 10.1016/0165-0114(94)90162-7

[4] A. George and P. Veeramani, On some results of analysis for fuzzy metric spaces. Fuzzy sets and systems, 90(3), 365-368 (1997), doi 10.1016/S0165-0114(96)00207-2

[5] V. Gregori, S. Morillas, B. Roig, and A. Sapena, "Fuzzy averaging filter for impulse noise reduction in colour images with a correction step", Journal of Visual Communication and Image Representation, 55, 518-528 (2018). doi 10.1016/j.jvcir. 2018.06.025.

[6] V. Gregori, S. Morillas, and A. Sapena, "Examples of fuzzy metrics and applications", Fuzzy sets and systems, 170(1), 95-111 (2011). doi 10.1018 /f.fss. 2010.10 .019

[7] V. Gregori and S. Romaguera, "Some properties of fuzzy metric spaces", Fuzzy Sets Syst. 115(3) , 485-489 (2000). doi 10.1016/S0165-0114(98)00281-4

[8] D. Karaklić, Lj. Gajić, N.M. Ralević, "Some Fixed Point Results in a Strong Probabilistic Metric Spaces", Filomat 33(8), 2201-2209 (2019). doi 10.2298 /FIL1908201K

[9] E.P. Klement, R. Mesiar, E. Pap, Triangular Norms. Kluwer Academic Publishers, Dordrecht (2000).

[10] G.J. Klir, and B. Yuan, Fuzzy sets and fuzzy logic: Theory and Applications. Prentice Hall, New Jersey (1995).

[11] I. Kramosil, and J. Michálek, "Fuzzy metrics and statistical metric spaces", Kybernetika, 11(5), 326-334 (1975).

[12] K. Menger, "Statistical metrics", Proc. Nat. Acad. of Sci. U.S.A., 28(12), 535-537 (1942).

[13] S. Morillas, V. Gregori, G. Peris-Fajarnes, and P. Latorre, "A fast impulsive noise color image filter using fuzzy metrics", Real-Time Imaging, 11(5-6), 417-428 (2005). doi 10.1016 /j.rti. 2005.06.007

[14] S. Morillas, V. Gregori, G. Peris-Fajarnes, and P. Latorre, "A New Vector Median Filter Based on Fuzzy Metrics". in: Kamel, M., Campilho, A. (Eds.). Image Analysis and Recognition - ICIAR2005, Lecture Notes in Computer Science, 3656, Springer-Verlag, Berlin, Heidelberg, 81-90 (2005). doi 10.1117/1.2767335

[15] S. Morillas, V. Gregori, G. Peris-Fajarnes, and A. Sapena, "New adaptive vector filter using fuzzy metrics, Journal of Electronic Imaging, 16(3), 033,007:1-15 (2007). doi 10.1116/1.2767335

[16] N.D. Narvekar and L.J. Karam, "An Improved No-Reference Sharpness Metric Based On The Probability Of Blur Detection", in: Conference Proceedings 2009 International Workshop on Video Processing and Quality Metrics or Consumer Electronics (VPQM) (2010).

[17] N. Ralević, S. Dukić, and D. Karaklić, Fazi metrike i primene u otklanjanju šuma na slici, The Fourth Mathematical Conference of the Republic of Srpska, Trebinje, Republika Srpska, 6 and 7 June 2014, Vol. II, Fakultet za proizvodnju i menadžment Trebinje, Univerzitet u Istocnom Sarajevu University of East Sarajevo, Mathematical Society of the Republic of Srpska, str. 101-109, ISBN 978-99976-600-4-6.

[18] N. M. Ralević and D. Karaklić, "Fazi rastojanja", META 2016, The First Conference on Mathematics in Engineering: Theory and Applications, Faculty of Technical Sciences, March 4$6^{\text {th }}$, 2016, Novi Sad, Serbia, str. 134-141, ISBN:978-86-7892-800-0.

[19] N. M. Ralević, D. Karaklić, and N. Pištinjat, "Fuzzy metric and its applications in removing the image noise", Soft Computing, 23(22), 12049-12061 (2019). doi 10.1007/s00500-01903762-5

[20] B. Smolka, M. Szczepanski, K. N. Plataniotis, and A. N. Venetsanopoulos, "On the fast modifyed vector median filter", Canadian Conference on Electrical and Computer Engineering, 1315-1319 (2001). doi 10.1109 /CCECE. 2001.93.23636

[21] Z. Wang, A.C. Bovik, "A universal image quality index", IEEE Signal Processing Letters, 9(3), 81-84 (2002). doi 10.1109/97.995823

Received May 20, 2020 